\title{
1020 MANEUVERING ON BENDS
}

A G Hemasiri, J Edirisinghe* Correspondence to Department of Civil Engineering, University of Peradeniya, Peradeniya 20400, Sri Lanka

10.1136/ip.2010.029215.1020

Driver ability is one of the key factors to improve road safety situation. The driver should be aware about the capacity of the 


\section{IP Safety 2010 abstracts}

vehicle, of the road and many other factors to drive a vehicle safely on roads. This image shows an accident due to driving high speed on a bend. 